\title{
Study on integrated scheduling method of networked cascade control system with double-sampling rate
}

\author{
Liu Dianting ${ }^{1, a}$, Zhang Xiaoyu ${ }^{1, b^{*}}$ \\ 1. College of Mechanical and Control Engineering, Guilin University of Technology, Guangxi 541004, \\ China \\ aliudian@glite.edu.cn,b165303801@qq.com
}

Keywords: comprehensive scheduling strategy; network cascade control system; sampling rate; Abstract: For network cascade control system with double-sampling rate, the paper proposes a new comprehensive scheduling strategy. In the comprehensive scheduling strategy, the variable sampling period algorithm is used in inner-loop and the forecast values are calculated by the cubic exponential differential smoothing method. The dead scheduling approach is used in outer-loop and the dead range adjustment algorithm is analyzed. Finally, an example is presented to test the validity and effectiveness of the comprehensive proposed in this paper.

\section{Introduction}

The network control system (NCSs) is characterized that the control information is exchanged through the network [1,2]. If the controlled object is connected via a network which have different sampling rate, it is called multi-rate NCSs. Network cascade control system is a special multi-rate NCSs, which have inner-loop and outer-loop.

Currently, the studies of NCS scheduling have mainly TOD dynamic scheduling [3,5], Variable sampling period scheduling [6,8], Dead scheduling [9,11]. But these methods can't adapt to network cascade control system with double-sampling rate, and its study of scheduling is less, now.

The comprehensive scheduling of network cascade control system is studied in the paper, the variable sampling period scheduling is used in the inner-loop, and the dead scheduling is used in the outer-loop. The simulation proved that the method is effective on network cascade control system with double-sampling rate.

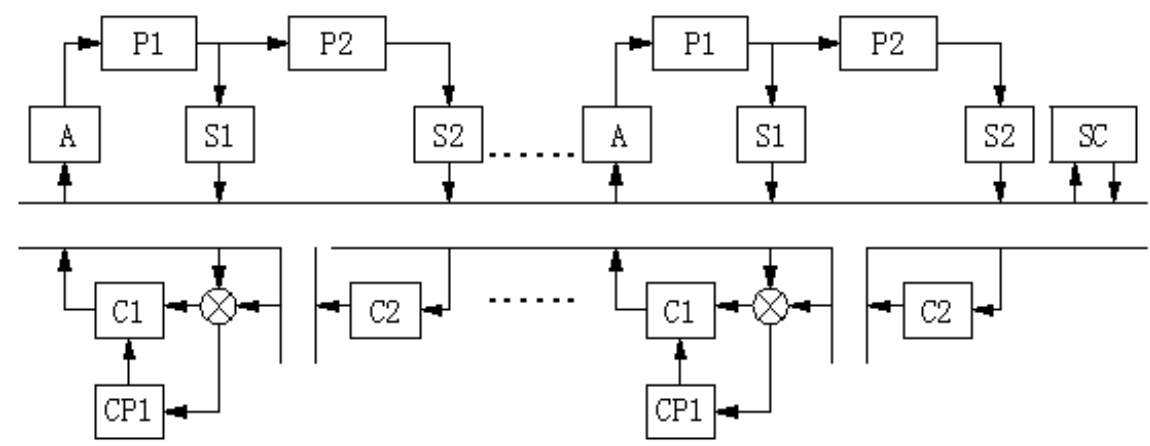

Figure 1 the structure of network cascade control system with double-sampling rate

\section{The comprehensive scheduling program}

As shown in figure 1, P1 is the controlled object 1, P2 is the controlled object 2, $\mathrm{A}$ is the actuator, $\mathrm{S} 1$ is the sensor $1, \mathrm{~S} 2$ is the sensor 2, $\mathrm{C} 1$ is the controller $1, \mathrm{C} 2$ is the controller 2, CP1 is the dead District compensator, $\mathrm{SC}$ is the scheduler. $\mathrm{P} 1, \mathrm{~S} 1, \mathrm{C} 1$, and $\mathrm{A}$ is consist of inner-loop, P1, P2, S2, C2, $\mathrm{C} 1$, and $\mathrm{A}$ is consist of inner-loop.

The system has the following assume: 1) sensors are time-driven, controllers are event-driven. 2) The sampling period of inner-loop $T_{1}$ is less than the outer-loop $T_{2}$, and $j_{1}$ and $j_{2}$ are monitoring cycles about the inner-loop and outer-loop. 


\section{Inner-loop scheduling}

\section{Network utilization rate and execution time}

$U_{1}^{i}$ and $\hat{U}_{1}^{i}$ is network utilization rate in the actual and predicted; $\dot{U}_{1}^{i(1)} \dot{U}_{1}^{i(2)}$ and $\dot{U}_{1}^{i(3)}$ is respective first order, second order and third order smoothed value, $\alpha$ is the smoothing factor which is the 0.1 to 0.9 . The forecasting model of network utilization rate in the inner-loop is

Which

$$
\hat{U}_{1}^{i}\left(j_{1}+1\right)=E_{U}+F_{U}+G_{U}
$$

$$
\begin{aligned}
& E_{U}=3 \dot{U}_{1}^{i(1)}\left(j_{1}\right)-3 \dot{U}_{1}^{i(2)}\left(j_{1}\right)+\dot{U}_{1}^{i(3)}\left(j_{1}\right) \\
& F_{U}=\frac{\alpha}{2(1-\alpha)}\left[(6-5 \alpha) \dot{U}_{1}^{i(1)}\left(j_{1}\right)-2(5-4 \alpha) \dot{U}_{1}^{i(2)}\left(j_{1}\right)+(4-3 \alpha) \dot{U}_{1}^{i(3)}\left(j_{1}\right)\right] \\
& G_{U}=\frac{\alpha^{2}}{2(1-\alpha)^{2}}\left[\dot{U}_{1}^{i(1)}\left(j_{1}\right)-2 \dot{U}_{1}^{i(2)}\left(j_{1}\right)+\dot{U}_{1}^{i(3)}\left(j_{1}\right)\right] \\
& \dot{U}_{1}^{i(1)}\left(j_{1}\right)=\alpha \sum_{n=0}^{\infty}(1-\alpha)^{n} U_{1}^{i}\left(j_{1}-n\right) \\
& \dot{U}_{1}^{i(2)}\left(j_{1}\right)=\alpha \sum_{n=0}^{\infty}(1-\alpha)^{n} \dot{U}_{1}^{i(1)}\left(j_{1}-n\right) \\
& \dot{U}_{1}^{i(3)}\left(j_{1}\right)=\alpha \sum_{n=0}^{\infty}(1-\alpha)^{n} \dot{U}_{1}^{i(2)}\left(j_{1}-n\right)
\end{aligned}
$$

The execution time of forecast is similar to Network utilization rate of forecast.

$$
\hat{C}_{1}^{i}\left(j_{1}+1\right)=E_{C}+F_{C}+G_{C}
$$

\section{The distribution of the inner-loop network utilization rate}

At first, the minimum network utilization rate $U_{1 \min }^{i}$ is ensured in inner-loop[6]. The utilization rate in $j_{1}$ monitoring period is

$$
\bar{U}_{1}^{i}\left(j_{1}\right)=U_{1 \min }^{i}\left(j_{1}\right)+q^{i}\left[\hat{U}\left(j_{1}+1\right)-\sum_{i=1}^{n} U_{\min }^{i}\left(j_{1}\right)\right]
$$

Which $U_{1 \text { min }}^{i}\left(j_{1}\right)=\sum_{i=1}^{n} \hat{C}^{i}\left(j_{1}\right) / \hat{T}_{\text {max }}^{i}, \hat{T}_{\text {max }}^{i}$ is the largest sampling period in the inner loop, the demand value of the inner network resources is $q^{i}=I A E_{1}^{i} / \sum_{r=1}^{N} I A E_{1}^{r}(r=1, \ldots, N)$.

At the $j_{2}$ monitoring period, the inner-loop sampling time have two cases: (1) the outer-loop and inner-loop have different sample time; (2) the inner-loop and outer-loop of sample time is same.

$d^{i}\left(j_{2}\right)$ is the difference value of inner and outer in the $i$ loop at the $j_{2}$ monitoring cycles.

$$
d^{i}\left(j_{2}\right)=\left[T_{1}^{i}\left(j_{2}\right)+\ldots+T_{1}^{i}\left(j_{2}\right)+d^{i}\left(j_{2}-1\right)\right]-T_{2}^{i}\left(j_{2}\right)
$$

The network utilization of the $j_{2}$ monitoring period are:

(1) If $d^{i}\left(j_{2}\right) \neq 0$, which is satisfied the conditions (1), the inner-loop of the network utilization is $\bar{U}_{1}^{i}(t)=\bar{U}_{1}^{i}\left(j_{1}\right), t=j_{1}$ or $j_{2}$

(2) If $d^{i}\left(j_{2}\right)=0$, which is satisfied the conditions (2), the assigned value of inner-loop network utilization is $\bar{U}_{1}^{i}(t)=\left\{\begin{array}{c}\bar{U}_{1}^{i}\left(j_{1}\right), t=j_{1} \\ U_{1 \text { min }}^{i}\left(j_{2}\right), t=j_{2}\end{array}\right.$

\section{Inner-loop sampling period}

The sampling period $T_{1}^{i}$ of the inner-loop have 


$$
T_{1}^{i}(t)=\hat{C}_{1}^{i}(t) / \bar{U}_{1}^{i}(t)=\left\{\begin{array}{c}
\hat{C}_{1}^{i}\left(j_{1}\right) / \bar{U}_{1}^{i}\left(j_{1}\right), t=j_{1} \\
\hat{C}_{1}^{i}\left(j_{2}\right) / \bar{U}_{1}^{i}\left(j_{2}\right), t=j_{2}, d\left(j_{2}\right) \neq 0 \\
\hat{C}_{1}^{i}\left(j_{2}\right) / U_{1 \text { min }}^{i}\left(j_{2}\right), t=j_{2}, d\left(j_{2}\right)=0
\end{array}\right.
$$

The inner-loop sampling period is bounded and varied, which is $T_{1}^{i} \in\left[T_{1 \min }^{i}, T_{1 \max }^{i}\right]$, $T_{1 \min }^{i}=C_{1 \min }^{i} / U_{\max }$ and $2 D^{i} / R \leq T_{1 \max }^{i} \leq 2 \pi / 10 F . C_{1 \min }^{i}$ is the execution time of $i$ loop without packet collisions, $C_{1 \min }^{i}=2 L^{i} / B ; L^{i}$ is the length of the packet in the $i$ loop; B is the transfer rate. $U_{1 \max }^{i}$ is the maximum network utilization of the $i$ inner-loop. The minimum throughput of the network is $\mathrm{R}$, the length of the $i$ inner-loop message is $D^{i}$, the maximum frequency of the system is $\mathrm{F}$.

\section{Outer-loop scheduling}

\section{Dead criterion}

The dead scheduling algorithm

$$
\Delta y_{p 2}^{i}\left(j_{2}\right)=\left|y_{p 2}^{i}\left(j_{2}\right)-y_{p 2}^{i}\left(j_{2}-1\right)\right| \leq d^{i}
$$

$y_{p 2}^{i}$ is the output of the sensor 2 in the i-loop. $d^{i}$ is the dead zone value in the $i$ loop.

At the outer-loop sampling time, sensor 2 determined whether information meets Dead criterion. If it meets Dead criterion, the sensor 2 stops sending signals.

\section{The dead range adjustment algorithm}

The dead range adjustment algorithm is used to adjust the value of the dead zone. In [11], the dead value $d^{i}$ is more than 0.03 , the performance of the system is deterioration. $M_{u}^{i}$ is the missing rate in the $d^{i}=0.03, M_{m}^{i}$ is the missing rate when the $Q_{2}^{i}$ is maximum, $M^{i}$ is the missing rate in dead value $d^{i}, M^{i}=\left[M_{u}^{i}, M_{m}^{i}\right]$

The dead range adjustment algorithm is

$$
d^{i}\left(j_{2}\right)=d^{i}\left(j_{2}-1\right)+\Delta d^{i}\left(j_{2}\right)
$$

Which $\Delta d^{i}\left(j_{2}\right)=K_{p}\left[r\left(j_{2}\right)-r\left(j_{2}-1\right)\right]+K_{1} e\left(j_{2}\right)+K_{D}\left[r\left(j_{2}\right)-2 r\left(j_{2}-1\right)+r\left(j_{2}-2\right)\right], K_{p}, K_{1}$ and $K_{D}$ is determined by the simulation. The adjustment value $r\left(j_{2}\right)$ is selected as

$$
r\left(j_{2}\right)=\left\{\begin{array}{c}
M_{u}^{i}, M_{i}\left(j_{2}\right) \in\left[0, M_{u}^{i}\right] \\
0, M_{i}\left(j_{2}\right) \in\left[M_{u}^{i}, M_{m}^{i}\right] \\
M^{i}, M_{i}\left(j_{2}\right) \in\left[M_{m}^{i},+\infty\right]
\end{array}\right.
$$

\section{Simulation}

TureTime1.5 is used to simulate the system. Three systems have same parameters. The controlled object 1

$$
A_{1}=\left[\begin{array}{ccc}
-0.2 & -1 & -0.1 \\
1.2 & 0 & 0 \\
0 & 1 & 0
\end{array}\right] B_{1}=\left[\begin{array}{lll}
1 & 0 & 0
\end{array}\right]^{T} C_{1}=\left[\begin{array}{lll}
0 & 0 & 0.135
\end{array}\right]^{T}
$$

The controlled object 2

$$
A_{2}=\left[\begin{array}{cc}
-0.2 & -0.011 \\
5 & 0
\end{array}\right] B_{2}=\left[\begin{array}{ll}
1 & 0
\end{array}\right]^{T} C_{2}=\left[\begin{array}{ll}
0 & 0.011
\end{array}\right]^{T}
$$

The system have $K_{1}=-0.19, K_{2}=0.35$. The initial sampling period are $T_{1}=1 \mathrm{~s}$ and $T_{2}=3 \mathrm{~s}$. The maximum sampling period of inner-loop is $T_{1 \max }=10 \mathrm{~s}$, the network bandwidth is $800 \mathrm{~b} / \mathrm{s}$. the simulation time is $300 \mathrm{~s}$. The input is the square wave of $0.008 \mathrm{~Hz}$. 

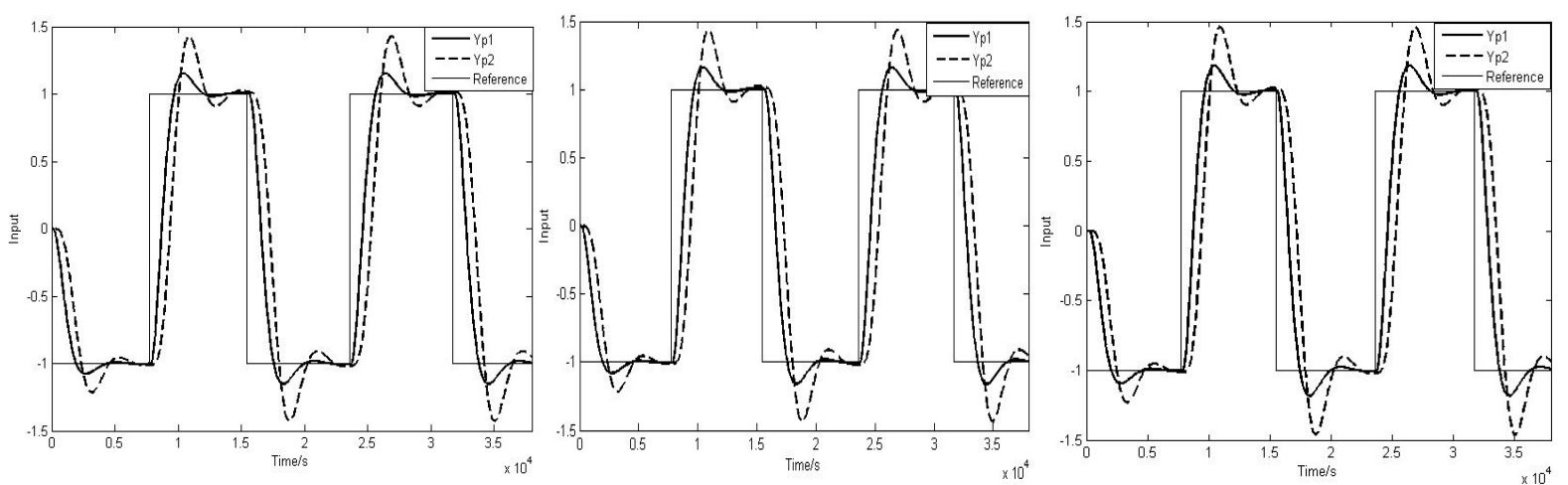

Figure 2 the input/output response of loop1/ loop2/ loop3 in the comprehensive strategy

\section{Conclusions}

For network cascade control system with double-sampling rate, the comprehensive scheduling strategy is proposed in the paper. The variable sampling period scheduling algorithm is used in the inner-loop, the sampling period is forecasted by the cubic exponential smoothing method. The dead zone scheduling is adopted in outer-loop. The simulation shows that the comprehensive scheduling strategy can regulate multi-loop system to keep the performance.

\section{Acknowledgements}

This work has been supported by National Natural Science Foundation of China (Grant No. 51165004), and Key Research Project of Guangxi Higher Education (Grant No.201202ZD048), and Doctoral Fund of Guilin University of Technology in 2012, and Innovation project of Guangxi Graduate Education (Grant No. YCSZ2014153)

\section{Reference}

[1] C. Zhou, M. L. Du, Q. W. Chen, Co-design of dynamic scheduling and H-infinity control for networked control system, J. Applied Mathematics and Computation. 218 (2012) 10767-10775

[2] D. S. Kim, D. H. Choi, P. M. Patra, Real-time scheduling method for networked discrete control systems, J. Control Engineering Practice. 17 (2009) 564-570

[3] E. Tian, X. Zhao, Robust control for uncertain networked systems with communication constraints, J. Journal of the Franklin Institute. 350 (2013) 1926-1943

[4] Y. L. Sun, H. Nael. E. I. Farra, Resource aware quasi decentralized control of networked process systems over wireless sensor networks, J. Chemical Engineering Science. 69(2012)93-106

[5] X. H. Yi, B. Li, Research on variable sampling period algorithm in networked control system, J. Journal of Beijing Jiao-tong University. 34(2012)135-141

[6] X. X. Dai, A. L. Liu, Application of a new variable sampling strategy in uncertain load NCS, J. Journal of East China University of science and technology. 38(2012)95-102

[7] Y. M. He, S. L. Wei, Reasearch of co-design feedback scheduling strategy for NCSs based on Grey Prediction, J. Computer Measurement and Control, 20(2012)3232-3235

[8] Z. N. Gao, R. H. Xie, $H \infty$ control of wireless networked control systems with signal difference-based deadband scheduling, J. Control and Decision. 27(2012)1301-1308

[9] J. D. Wang, J. S. Yu, An enhanced deadband feedback scheduling approach in networked control system, J. Journal of East China University of science and technology. 34(2008)579-584

[10] Y. C. Tian, D. Levy, Compensation for control packet dropout in networked control systems, J. Information Sciences. 178(2008)1263-1278

[11] Q. Zhang, J. S. Yu, Deadband feedback scheduling based on PID algorithm in networked control system, J, Control and Instruments in Chemical Industry. 35(2008)14-17

[12] Y. Shen, B. Guo, Variable-sampling period intelligent dynamic scheduling for networked control systems, J. Journal of SiChuan University. 42(2010)162-167 
[13] W. G. Shi, Y. Tang, C. Shao, The network control system scheduling algorithm for recursive and variable sampling period, J. Control and Instruments in Chemical Industry. 38(2011)1299-1302. 